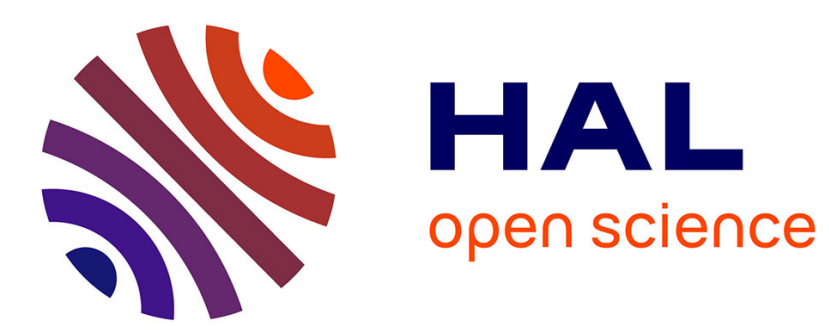

\title{
On Cancellation of Transactions in Bitcoin-like Blockchains
}

Önder Gürcan, Alejandro Ranchal Pedrosa, Sara Tucci-Piergiovanni

\section{To cite this version:}

Önder Gürcan, Alejandro Ranchal Pedrosa, Sara Tucci-Piergiovanni. On Cancellation of Transactions in Bitcoin-like Blockchains. 26th International Conference on COOPERATIVE INFORMATION SYSTEMS, Oct 2018, La Valette, Malta. cea-01867357

\section{HAL Id: cea-01867357 https://hal-cea.archives-ouvertes.fr/cea-01867357}

Submitted on 4 Sep 2018

HAL is a multi-disciplinary open access archive for the deposit and dissemination of scientific research documents, whether they are published or not. The documents may come from teaching and research institutions in France or abroad, or from public or private research centers.
L'archive ouverte pluridisciplinaire $\mathbf{H A L}$, est destinée au dépôt et à la diffusion de documents scientifiques de niveau recherche, publiés ou non, émanant des établissements d'enseignement et de recherche français ou étrangers, des laboratoires publics ou privés. 


\title{
On Cancellation of Transactions in Bitcoin-like Blockchains
}

\author{
Önder Gürcan ${ }^{1}$ and Alejandro Ranchal Pedrosa ${ }^{1,2,3}$ \\ and Sara Tucci-Piergiovanni ${ }^{1}$ \\ ${ }^{1}$ CEA LIST, Point Courrier 174, Gif-sur-Yvette, F-91191 France \\ ${ }^{2}$ LIP6 - Sorbonne Université, Paris, France \\ 3 EIT Digital, Stockholm, Sweden \\ \{Onder.Gurcan, Alejandro.Ranchal-Pedrosa, Sara.Tucci\}@cea.fr
}

\begin{abstract}
Bitcoin-like blockchains do not envisage any specific mechanism to avoid unfairness for the users. Hence, unfair situations, like impossibility of cancellation of transactions explicitly or having unconfirmed transactions, reduce the satisfaction of users dramatically, and, as a result, they may leave the system entirely. Such a consequence would impact significantly the security and the sustainability of the blockchain. Based on this observation, in this paper, we focus on explicit cancellation of transactions to improve the fairness for users. We propose a novel scheme with which it is possible to cancel a transaction, whether it is confirmed in a block or not, under certain conditions. We show that the proposed scheme is superior to the existing workarounds and is implementable for Bitcoin-like blockchains.
\end{abstract}

\section{Introduction}

Bitcoin, introduced by Satoshi Nakamoto [13], is the core of decentralized cryptocurrency systems. Participants following this protocol can create together a distributed, economical, social and technical system where anyone can join and leave. User participants create and broadcast transactions across the network for being confirmed. Miner participants try to confirm them as a block by solving a computational puzzle (mining). Successful miners broadcast their block to the network to be chained to the blockchain, being rewarded for their success. In addition, all participants validate all data (transactions and blocks) broadcast across the network. It is a very attractive technology, since it maintains a public, immutable and ordered log of transactions which guarantees an auditable ledger, accessible by anyone.

The security and sustainability of blockchains, however, are not trivial and require increased participation, since each participant validates the diffused data, and keeps a replica of the entire blockchain. Participants consider worthwhile to join and stay in the system over time only if they find it fair [7]. Miner participants find the system fair if they are able to create blocks as they expected, and user participants find the system fair if they manage to cancel their transactions 
and/or their transactions are confirmed as they expected. Considering miners, several formal studies have been conducted so far $[6,5,18,15]$, concluding that Bitcoin-like blockchains are not promoting participation of miners.

In [7], it has been for the first time shown that Bitcoin-like blockchains are unfair for user participants. It has also been discussed that for the time being it is not possible to explicitly cancel a transaction. There are only some workarounds that a user can try in order to cancel its transaction. One of these workarounds consist of trying to replace an old transaction with the new one with higher fees (Replace-By-Fee $\left.(\mathrm{RBF})^{4}\right)$. This way, it is possible to create another transaction attempting to spend the same inputs but sending the money to the issuer itself. This is a workaround for cancellation of unconfirmed transactions by implicitly marking them to prevent their further use. However, once a transaction is confirmed, there is no workaround to reverse the situation. Since double spending is not allowed, the miners will not put both transactions in their blocks. However, there is no guarantee that the double spending transaction will arrive to the miners before the confirmation of the first transaction inside a block. Since it is like that, once a user decides to issue a transaction, $\mathrm{s} / \mathrm{he}$ can never abandon this decision. Considering that a user is a rational agent that aims to maximize its utility by choosing to perform the actions with the optimal expected outcomes [17], this implies the utility is going to be minus infinity [7]. In the decision theory terms, this would mean assuming a user having an infinite interest on a transaction, which is hard to assume in realistic settings.

To this end, we propose a novel scheme where it is possible to cancel a transaction by rolling back its state whether it is confirmed in a block or not under certain conditions. This way, the utility of user agents can be maximized and, consequently, their willingness to leave the system decreases.

The contributions of this paper are as follows:

- A novel scheme based on a novel type of transaction that enables explicit cancellation of transactions.

- The implementation of such mechanism in Bitcoin-like blockchains.

The remainder of this paper is organized as follows. Section 2 gives the related work. Section 3 provides a formalization of the existing Bitcoin-like blockchain data structure considered in this paper. Section 4 provides a high-level Bitcoinlike blockchain protocol description as a rational multi-agent model where agents are using the aforementioned data structure. The proposed scheme for cancellation of transactions is presented in Section 5. Section 6 presents an analysis of the proposed scheme and, finally, the discussion and conclusions are provided in Sections 7 and 8, respectively.

\footnotetext{
${ }^{4}$ https://en.bitcoin.it/wiki/Replace_by_fee, last access on 16 July 2018.
} 


\section{Related Work}

This study is based on our previous study on user (nodes that do not participate to the mining) fairness in blockchain systems [7]. The closest works in blockchain systems to our study are $[8,4,1,12]$.

Herlihy and Moir in [8] study the user fairness and consider as an example the original Tendermint ${ }^{5}[3,9]$. The authors discussed how processes with malicious behaviour can violate fairness by choosing transactions [4] then they propose modifications to the original Tendermint to make those violations detectable and accountable. Helix [1] and HoneyBadgerBFT [12], on the other hand, attempt to design consensus protocols focusing on assuring a degree of fairness among the users by being resilient to transaction censorship where initially encrypted transactions are included in blocks, and only after their order is finalized, the transactions are revealed.

Another notion of fairness applied to the user side concerns the fair exchange in the e-commerce context [2] which is extended to the Bitcoin-Like scenario in [10] more in the sense that if there are two players performing an exchange then either both of them get what they want or none of them.

\section{Blockchain Model}

We model a blockchain ledger as a dynamic, append-only tree $B=\left\{b_{0} \longleftarrow\right.$ $\left.b_{1} \stackrel{\#_{1}}{\longleftarrow} \ldots \stackrel{\#_{l-1}}{\longleftarrow} b_{l}\right\}$ where each block $b_{i}(0<i \leq l)$ contains a cryptographic reference $\#_{i-1}$ to its previous block $b_{i-1}, l=|B|$ is the length of $B, b_{0}$ is the root block which is also called the genesis block, $b_{l}$ is the furthest block from the genesis block which is referred to as the blockchain head, $h=\left|b_{i}\right|$ is the height of $b_{i}$ (the length of the path from $b_{i}$ block to $\left.b_{0}\right)^{6}$ and $d=|B|-\left|b_{i}\right|$ is the depth of $b_{i}$ (the length of the path from $b_{i}$ block to $b_{l}$ ).

A block $b_{i-1}$ can have multiple children blocks, which causes the situation called a fork. The main branch is then defined as the longest path $l$ from any block to $b_{0}$ and is denoted as $B^{\star}$ where $\left|B^{\star}\right|=l$ and $B^{\star} \subseteq B$ such that $\left|B^{x}\right|<$ $\left|B^{\star}\right|$ for all branches $B^{x} \subset B$ where $B^{x} \neq B^{\star}$. All branches other than the main branch are called side branches. If at any time, there exists more than one longest path with a length $l$ (i.e. there are multiple heads), the blockchain ledger $B$ is said to be inconsistent and thus $B^{\star}=\emptyset$. This situation disappears when a new block extends one of these side branches and creates $B^{\star}$. The blocks on the other branches are discarded and referred as stale blocks.

\subsection{Block Model}

We denote a block as $b_{i}=\left\langle\mathrm{h}_{i}, \Psi_{i}\right\rangle$ where $\mathrm{h}_{i}$ is the block header and $\Psi_{i}$ is the block data. The block data $\Psi_{i}$ contains a set of transactions organized as a Merkle tree

\footnotetext{
${ }^{5}$ Jae Kwon and Ethan Buchman. Tendermint. https://tendermint.readthedocs. io/en/master/specification.html, last access on 25 July 2018.

${ }^{6}$ For each transaction $t x$ inside $b_{i}$, the block height $|t x|$ is the equal to $h$ also.
} 
[11]. The set of transactions $\theta_{m}$ are selected by the miner from its memory pool. Here it is important to note that, blocks have limited sizes ${ }^{7}$ and thus the total size of the selected transactions can not exceed this limit ${ }^{8}$.

\subsection{Transaction Model}

We model a transaction as $t x=\langle I, O\rangle$ where $I$ is a list of inputs $(I \neq \emptyset)$ and $O$ is a list of outputs $(O \neq \emptyset)$. Each input $i \in I$ references to a previous unspent output for spending it. Each output then stays as an Unspent Transaction Output (UTXO) until an input spends it. If an output has already been spent by an input, it cannot be spent again by another input (no double spending). We model the outputs as $o_{i}=\left\langle s_{i}, \Phi_{o_{i}}\right\rangle$ where $s_{i}$ is a set of tuples $s_{i}=\left\{\left(n_{i}\right.\right.$, cond $\left.\left.s_{n_{i}}\right)\right\}$ that define the conditions conds $s_{n_{i}}$ for the receiver $n_{i} \in N$ to become owner of the coin $\Phi_{o_{i}}\left(\Phi_{o_{i}} \geq 0\right)$. The input that wants to spend the particular coin must satisfy at least one list of conditions $\operatorname{conds}_{n_{i}}$, since an output (a coin) might be spendable by two different receivers $n_{i}, n_{j} \in N$ independently, although it will ultimately be spent by only one, on a first-come, first-served basis. All inputs of a transaction have to be spent in that transaction and the total input coins $\mathbb{\phi}_{I}$ has to be greater than or equal to the total output coins $\Phi_{O}$. The fee $f_{t x}$ of a transaction $t x$ is then modeled as $f_{t x}=\phi_{I}-\phi_{O}$. Depending on the fee to be paid, if there are still some coins left to be spent, the sender can add an output that pays this remainder to itself.

\section{Network Model}

In this section we provide a high-level Bitcoin protocol description as a rational multi-agent model $^{9}$ where rational agents chooses their actions/behavior with respect to their perceptions in order to maximize their utility.

We model the blockchain network as a dynamic directed graph $G=(N, E)$ where $N$ denotes the dynamic rational agent (vertex) set, $E$ denotes dynamic directed link (edge) set. A link $\langle n, m\rangle \in E$ represents a directed link $n \rightarrow m$ where $n, m \in N, n$ is the owner of the link and $n$ is the neighbor of $m$.

\subsection{Agent Model}

Each agent $n \in N$ has a list of its neighbors $N_{n}$ where $N_{n} \subseteq N$ and $\forall m \in$ $N_{n} \mid\langle n, m\rangle \in E$. An agent $n$ can communicate one or more of its neighbors by exchanging messages of the form $\langle n, m s g, d\rangle$ where $n$ is the sender, $m s g$ is the type and $d$ is the data contained. Using such messages, the (user) agents

\footnotetext{
7 The current maximum block size in Bitcoin is 1 MB. See https://bitcoin.org/en/ glossary/block-size-limit, last access on 13 July 2018.

8 Average block size for Bitcoin is given in https://blockchain.info/charts/ avg-block-size, last access on 13 July 2018.

9 This description is based on the system and rational models given in [7].
} 
issue transactions (by creating transactions messages and diffusing them to the network) to send coins to each other.

Each agent $n$ has a memory pool $\Theta_{n}$ in which it keeps unconfirmed transactions that have input transactions, an orphan pool $\bar{\Theta}_{n}$ in which they keep unconfirmed transactions that have one or more missing input transactions (orphan transactions) and a blockchain ledger $B_{n}$ in which they keep confirmed transactions where $\Theta_{n} \cap \bar{\Theta}_{n}=\emptyset, \Theta_{n} \cap B_{n}=\emptyset$ and $\bar{\Theta}_{n} \cap B_{n}=\emptyset$ always hold.

A (user) agent $n$ can turn to be a miner agent if it chooses to create blocks for confirming the transactions (mining) in its memory pool $\Theta_{m}$, and $n$ is said to be a miner node if it started mining but has not stopped yet. The set of miner agents is then denoted by $M$ where $M \subseteq N$. In order to mine, $n \in M$ has to solve a cryptographic puzzle (i.e. Proof of Work) using its hashing power. The successful miners are awarded by a fix amount of reward plus the totality of the transaction fees.

\subsection{Behavior Model}

A rational agent behaves according to its local perceptions and local knowledge, models uncertainty via expected values of variables or actions, and always chooses to perform the actions with the optimal expected outcome (among all feasible actions) for maximizing its utility [17]. Each rational agent $n \in N$ has a set of actions $A_{n}$ and a utility function $\mathcal{U}_{n}$. Using $A_{n}$ and $\mathcal{U}_{n}, n$ uses a decision process where it identifies the possible sequences of actions to execute. We call these sequences as rational behaviors of $n$ and denote as $\beta$. The objective of $n$ is to choose the behaviors that selfishly keep $\mathcal{U}_{n}$ as high as possible.

We model the utility function of a rational agent $n \in N$ as

$$
\mathcal{U}_{n}=u_{0}+\sum_{i=1}^{k} \mathcal{U}\left(\beta_{i}\right)
$$

where $u_{0}$ is the initial utility value, $k \geq 0$ is the number of behaviors executed so far and $\mathcal{U}\left(\beta_{i}\right)$ is the utility value of the behavior $\beta_{i}$. An agent $n \in N$ finds a system (i.e. the blockchain network) $G$ fair, if the total satisfaction of its expectations $\mathcal{U}_{n}$ is above a certain degree $\tau_{n}$ where $\tau_{n}<u_{0}$ [7].

A utility value $\mathcal{U}\left(\beta_{i}\right)$ can also be interpreted as the degree of satisfaction experienced by the realization of $\beta_{i}$. The utility value $\mathcal{U}\left(\beta_{i}\right)$ is calculated as

$$
\mathcal{U}\left(\beta_{i}\right)=\mathcal{R}\left(\beta_{i}\right)-\mathcal{C}\left(\beta_{i}\right)
$$

where $\mathcal{R}\left(\beta_{i}\right)$ is the overall reward gained and $\mathcal{C}\left(\beta_{i}\right)$ is the overall cost spent for the execution of $\beta_{i}$.

When an agent needs to choose a behavior for execution, it needs to calculate its expected utility value. The expected value $\mathcal{E}\left(\beta_{i}\right)$ depends on the probabilities of the possible outcomes of the execution of $\beta_{i}$. We model the expected value as 


$$
\begin{aligned}
\mathcal{E}\left(\beta_{i}\right) & =\sum_{j=1}^{m}\left(p_{j} \cdot \mathcal{U}\left(\beta_{i}^{j}\right)\right) \\
& =\sum_{j=1}^{m}\left(p_{j} \cdot\left(\mathcal{R}\left(\beta_{i}^{j}\right)-\mathcal{C}\left(\beta_{i}^{j}\right)\right)\right) \\
& =\sum_{j=1}^{m}\left(p_{j} \cdot \mathcal{R}\left(\beta_{i}^{j}\right)\right)-\sum_{j=1}^{m}\left(p_{j} \cdot \mathcal{C}\left(\beta_{i}^{j}\right)\right) \\
& =\mathcal{R}^{\mathcal{E}}\left(\beta_{i}\right)-\mathcal{C}^{\mathcal{E}}\left(\beta_{i}\right)
\end{aligned}
$$

where $m>0$ is the number of possible outcomes, $\mathcal{U}\left(\beta_{i}^{j}\right)$ is the utility value of the possible $j$ th outcome $\beta_{i}^{j}, p_{j}$ is the probability of this outcome such that $\sum_{j=1}^{m} p_{j}=1$, and $\mathcal{R}^{\mathcal{E}}\left(\beta_{i}\right)$ and $\mathcal{C}^{\mathcal{E}}\left(\beta_{i}\right)$ are the expected gain and the expected cost of $\beta_{i}$ respectively.

In the following, we list the user and miner agents behaviors important for this study ${ }^{10}$ conforming to the above description. To formalize the behaviors, we model a round based approach (like Garay et al. [6]) in which miner agents start creating a new block with at the beginning of the round and a round ends when a new block is successfully created by one of the miners. Both user and miner agents make their decisions on a roundly basis. This round-based model implicitly assumes that the block sent at at the end of the round is immediately delivered by all participants, i.e. communication delay is negligible with respect to block generation time.

Miner Agent Behaviors are as follows:

- Selecting transactions. When creating the next block, there is no required selection strategy and no known way to make any particular strategy required, but there are two transaction selection strategies popular among miners to include them into their blocks: (1) selecting the transactions with the highest fees to attempt to maximize the amount of fee income they can collect ${ }^{11}$. Since the size of blocks is limited, miners could decide to deliberately exclude an unconfirmed transaction that has already been received with a lower fee. This behavior would obviously delay the confirmation time of that transaction and affects its confirmation probability. (2) selecting the transactions with highest amount of coins moved to attempt to maximize the market value of the cryptocurrency [14]. Since the size of blocks is limited, miners could decide to deliberately exclude an unconfirmed transaction that has already been received with a lower amount. In this study, it is assumed that miners are using the 1st selection strategy and this behavior is modeled as $P(f)$ probability function.

$\overline{10}$ A detailed list of behaviors, along with their pseudo-codes, can be found in [7].

11 https://en.bitcoin.it/wiki/Transaction_fees, last access on 20 July 2018. 
User Agent Behaviors are as follows:

- Issuing transactions. We model issuing a transaction with a specific fee $f$ as with the action of the form issueTransaction $(b, \mathbb{\phi})$ where $b \in N$ is the receiver and $\phi$ is the amount of coins (Algorithm 1). For simplicity, it is assumed that a users agent has an ordered set of fees $\left\{f_{1}, f_{2}, \ldots, f_{k}\right\}$ to use. It is also assumed that $f_{i}<f_{i+1}$ where $0<i<k$ and $0 \leq P\left(f_{i}\right)<P\left(f_{i+1}\right) \leq 1$ where $P(f)$ is the probability of a transaction with a fee $f$ to be confirmed.

- Leaving because of unfairness. If at any time, an agent $a$ finds $G$ unfair $\left(\mathcal{U}_{a} \leq \tau_{a}\right)$, it may decide to leave $G$ if from its points of view it will not be possible to increase its overall utility above $\tau_{a}$ by calculating the expected values of its possible future behaviors. In other words, $a$ may decide to leave $G$ if $\mathcal{U}_{a}+\sum_{j=k}^{m} \mathcal{E}\left(\beta_{j}\right) \leq \tau_{a}$ where $\beta_{k}, \ldots, \beta_{m}$ are sufficiently enough desired future behaviors of $a$.

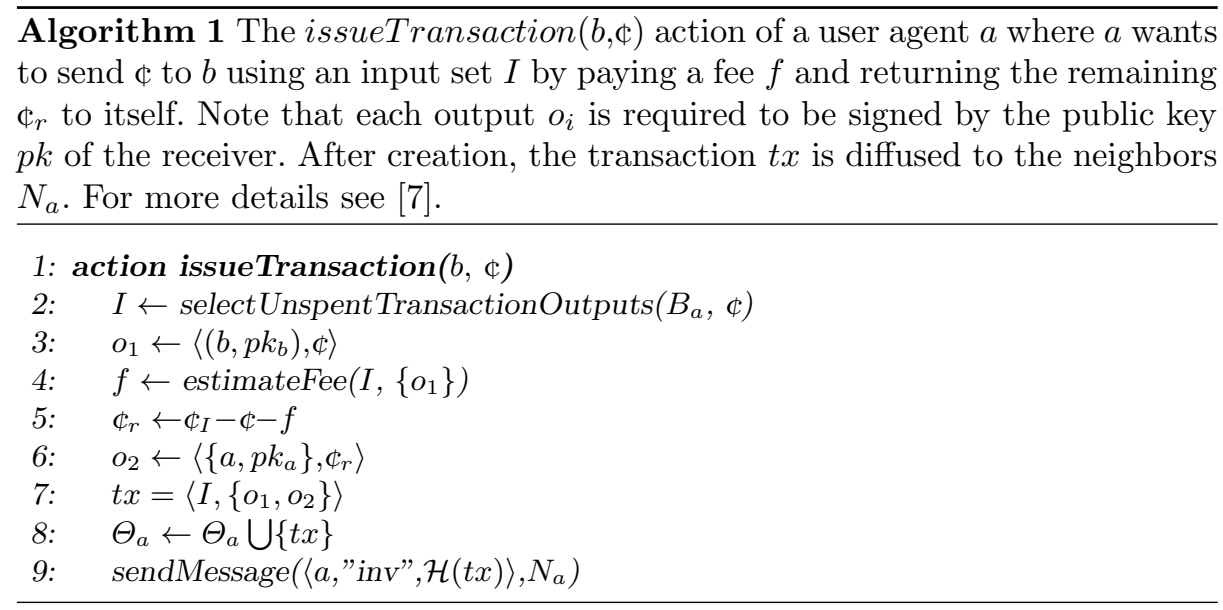

In the next section, we present our proposed improvements to allow cancellation of transactions.

\section{Cancellation of Transactions}

In this section, we improve the models given in Section 3 and Section 4 to enable cancellation of transactions. We first make necessary definitions (Section 5.1), then provide a new model for canceling transactions (Section 5.2) and finally provide user agent behaviors for canceling transactions (Section 5.3).

\subsection{Definition}

We define cancellation of transactions as intentionally marking a transaction by its issuer to prevent its further use, i.e. negating the effect of a transaction 
by its issuer and thus creating a new state as if the issuer of the transaction has never sent money to the recipients ${ }^{12}$. Here it should be emphasized that such cancellation can only be performed by the issuer of the transaction, since the receiver can always create another transaction that returns the money back to the original account and sign it. However, such a case is not considered as cancellation but as refunding.

\subsection{Cancellation-enabled Transaction Model}

In this subsection, we improve the transaction model given in Section 3.2 to allow cancellation of transactions, as defined in Section 5.1.

We consider that a transaction can be canceled by its issuer before a predefined cancellation timeout $r_{c}$. Such a condition impacts directly in the required amount of time to consider that a transaction is final ${ }^{13}$. We define a final transaction as a transaction that is not cancellable any more. However, it should be noted that this definition is relative to a branch of the blockchain. If a side branch that does not contain a transaction becomes longer than the current main branch that contains that transaction, technically that transaction is said to be rolled back, however this is different from canceling it.

Being $t x=\langle I, O\rangle$ a valid transaction issued by $n \in N$, a cancellation transaction txc is a transaction issued by $n$ that gives ownership of the coins back to the user $n \in N$ that issued $t x$, although perhaps in a different address. In reality, our proposed solution gives more freedom than this definition, as we discuss further on.

\subsection{Cancellation Behaviors of User Agents}

Consider a user agent $a \in N$ that issues a transaction $t x$ at round $r_{0}$ for sending $\Phi$ coins to user node $b \in N$, with a fee of $f_{t x}$, has an interest $\mathcal{I}$ on $t x$ and a waiting cost $C(\phi)$.

Assuming that $t x$ is confirmed at most at round $n$, the cumulative expected value $\mathcal{E}$ is:

$$
\mathcal{E}\left(\beta_{0}\right)=\sum_{r=1}^{n} \overline{P(f)}^{r-1} \cdot P(f) \cdot(\mathcal{I}-f)-\sum_{r=1}^{n} \overline{P(f)}^{r-1} \cdot C(\phi)^{r-1}
$$

where $\mathcal{R}\left(\beta_{0}\right)=(\mathcal{I}-f)$ and $\mathcal{C}\left(\beta_{0}\right)=C(\phi)$ (based on Equation 1 in [7]).

Now suppose user agent $a$ decides to cancel $t x$ at round $r_{1}\left(r_{0} \leq r_{1}<r_{c}\right)$. There are two possible user agent behaviors to cancel $t x$ :

- $\beta_{1}$ : Canceling with cancellation transaction,

\footnotetext{
${ }^{12}$ It is important to note that the fee paid to the miner is not considered.

13 This complies with previous usage of transactions that are 'non-final':https://bitcoin.stackexchange.com/questions/9165/ whats-are-non-final-transactions, last access on 13 July 2018.
} 
- Explicit cancellation mechanism for transactions that are even confirmed, proposed by us in this paper (see Algorithm 2).

- $\beta_{2}$ : Canceling with a Replace-By-Fee (RBF) transaction

- Implicit cancellation mechanism for only unconfirmed transactions, proposed by Bitcoin.

where user agents calculate the expected values considering the gain and the cost of each behavior as in [7]. For simplicity, and without loss of generality, we consider that fees are not changing per round.

$\boldsymbol{\beta}_{1}$ : Canceling with cancellation transaction User creates a transaction $t x c$ at round $r_{1}$ with a fee $f_{t x c}$, where $f_{t x c}$ has a value such that $t x c$ is supposed to be appended to the blockchain in less than round $r_{c}+r$, being $r$ the round in which $t x$ hits the blockchain.

The expected gain $\mathcal{R}^{\mathcal{E}}\left(\beta_{1}\right)$ of behavior $\beta_{1}$ is then as follows:

$$
\begin{aligned}
\mathcal{R}^{\mathcal{E}}\left(\beta_{1}\right)= & \left(\sum_{r=r_{0}}^{n}{\overline{P\left(f_{t x}\right.}}^{r-1} P\left(f_{t x}\right) \cdot\left(\sum_{s=r+1}^{r+r_{c}}{\overline{P\left(f_{t x c}\right)}}^{s-1-r} P\left(f_{t x c}\right)\right)\right) \\
& \cdot\left(I-f_{t x}-f_{t x c}\right) \\
= & \left(\sum_{r=1}^{n}{\overline{P\left(f_{t x}\right)}}^{r-1} P\left(f_{t x}\right) \cdot\left(\sum_{s=1}^{r_{c}}{\overline{P\left(f_{t x c}\right)}}^{s-1} P\left(f_{t x c}\right)\right)\right) \\
& \cdot\left(\mathcal{I}-f_{t x}-f_{t x c}\right)
\end{aligned}
$$

where $\mathcal{R}\left(\beta_{1}\right)=\left(\mathcal{I}-f_{t x}-f_{f t x c}\right)$, and $r$ and $s\left(r_{0}<r \leq s\right)$ are the rounds in which $t x$ and $t x c$ are appended to the blockchain, respectively. Note that, for $r_{1}<$ $r$, the transaction $t x c$ is orphan and thus it can be appended in the blockchain only at $r=s$ or $r<s$. Furthermore, if $r_{1}>s$, then the best option is to use this behavior (since RBF transactions cannot be used anymore). Therefore, we compare for cases where $r_{0} \leq r_{1} \leq r$. For further simplification, without loss of generality, we use $r_{0}=1$.

The series given in Equation 5 follow two nested geometric distributions $X \backsim$ $\left(p_{t x}\right), Y \backsim\left(p_{t x c}\right)$, with probabilities of success $p_{t x}=P\left(f_{t x}\right)$ and $p_{t x c}=P\left(f_{t x c}\right)$. Therefore, considering $x=q_{t x}=1-p_{t x}$ and $y=q_{t x c}=\left(1-p_{t x c}\right)$, we have the following solution to the series:

$$
\mathcal{R}^{\mathcal{E}}\left(\beta_{1}\right)=\left(\sum_{r=1}^{n} x^{r-1} p_{t x} \cdot\left(\sum_{s=1}^{r_{c}} y^{s-1} p_{t x c}\right)\right) \cdot\left(\mathcal{I}-f_{t x}-f_{t x c}\right)
$$

We can extract all constant values into $c=p_{t x} \cdot p_{t x c} \cdot\left(\mathcal{I}-f_{t x}-f_{t x c}\right)$, and consider the solution to

$$
g_{\beta_{1}}(n)=\sum_{r=1}^{n} x^{r-1} \cdot \sum_{s=1}^{r_{c}} y^{s-1} \cdot c=\sum_{r=0}^{n-1} x^{r} \cdot \sum_{s=0}^{r_{c}-1} y^{s} \cdot c
$$


As such, notice that, applying basics of geometric convergent series, the inner series $\sum_{s=0}^{r_{c}-1} y^{s}$ converges to $\frac{1-y^{r_{c}}}{(1-y)}$ and thus the outer one:

$$
\begin{aligned}
g_{\beta_{1}}(n) & =\frac{\left(1-x^{n}\right)\left(1-y^{r_{c}}\right)}{(1-x)(1-y)} \cdot(1-x)(1-y)\left(\mathcal{I}-f_{t x}-f_{t x c}\right) \\
& =\left(1-x^{n}\right)\left(1-y^{r_{c}}\right)\left(\mathcal{I}-f_{t x}-f_{t x c}\right)
\end{aligned}
$$

And thus, being the expected gain when $g_{\beta_{1}}(n)$ tends to infinity:

$$
\lim _{n \rightarrow \infty} g_{\beta_{1}}(n)=\left(1-y^{r_{c}}\right)\left(\mathcal{I}-f_{t x}-f_{t x c}\right)
$$

As for the cost resulted from the Time Value of Money (TVM $)^{14}$, both transactions use the same amount money, but we consider that the TVM remains for as long as the transaction is not fully canceled. As such, the expected cost of behavior $\mathcal{C}^{\mathcal{E}}\left(\beta_{1}\right)$ of $\beta_{1}$ is modeled as follows:

$$
\mathcal{C}^{\mathcal{E}}\left(\beta_{1}\right)=\sum_{r=1}^{\infty}{\overline{P\left(f_{t x}\right.}}^{r-1} \cdot\left(\sum_{s=r_{1}}^{r+r_{c}}{\overline{P\left(f_{t x c}\right)}}^{s-1-r_{1}} \cdot C\left(\phi_{t x}\right)^{s-1}\right)
$$

Where $C(\Phi)$ represents the waiting cost derived of the time value of money, per round. These series are identical to $g_{\beta_{1}}(n)$ applying $x=q_{t x}, y=q_{t x c} \cdot C\left(\phi_{t x}\right)$ and the constant $c$ by $d=C\left(\Phi_{t x}\right)^{r_{1}}$, therefore, we define $h_{\beta_{1}}(n)$ :

$$
h_{\beta_{1}}(n)=\sum_{r=1}^{n} x^{r-1} \cdot \sum_{s=1}^{r+r_{c}-r_{1}} y^{s-1} \cdot d=\sum_{r=0}^{n-1} x^{r} \cdot \sum_{s=0}^{r+r_{c}-r_{1}-1} y^{s} \cdot d
$$

For which the result is analogously obtained as for $g_{\beta_{1}}(n)$ (note that $|x y|<1$ to guarantee convergence):

$$
h_{\beta_{1}}(n)=\left(\frac{1-x^{n}}{(1-y)(1-x)}-y^{r_{c}-r_{1}} \cdot \frac{1-(x y)^{n}}{(1-y)(1-x y)}\right) \cdot d
$$

And thus, again, being the cost when $h_{\beta_{1}}(n)$ tends to infinity:

$$
\begin{aligned}
\lim _{n \rightarrow \infty} h_{\beta_{1}}(n) & =\left(\frac{1}{(1-y)(1-x)}-y^{r_{c}-r_{1}} \cdot \frac{1}{(1-y)(1-x y)}\right) \cdot d \\
& =\left(\frac{1}{\left(1-q_{t x c} C\left(\phi_{t x}\right)\right)\left(1-q_{t x}\right)}-\left(q_{t x c} C\left(\phi_{t x}\right)\right)^{r_{c}-r_{1}}\right. \\
& \left.\cdot \frac{1}{\left(1-q_{t x c} C\left(\phi_{t x}\right)\right)\left(1-q_{t x} q_{t x c} C\left(\phi_{t x}\right)\right)}\right) \cdot C\left(\phi_{t x}\right)^{r_{1}}
\end{aligned}
$$

\footnotetext{
${ }^{14}$ The concept that indicates that money available at the present time worths more than the identical sum in the future due to its potential earning capacity.
} 


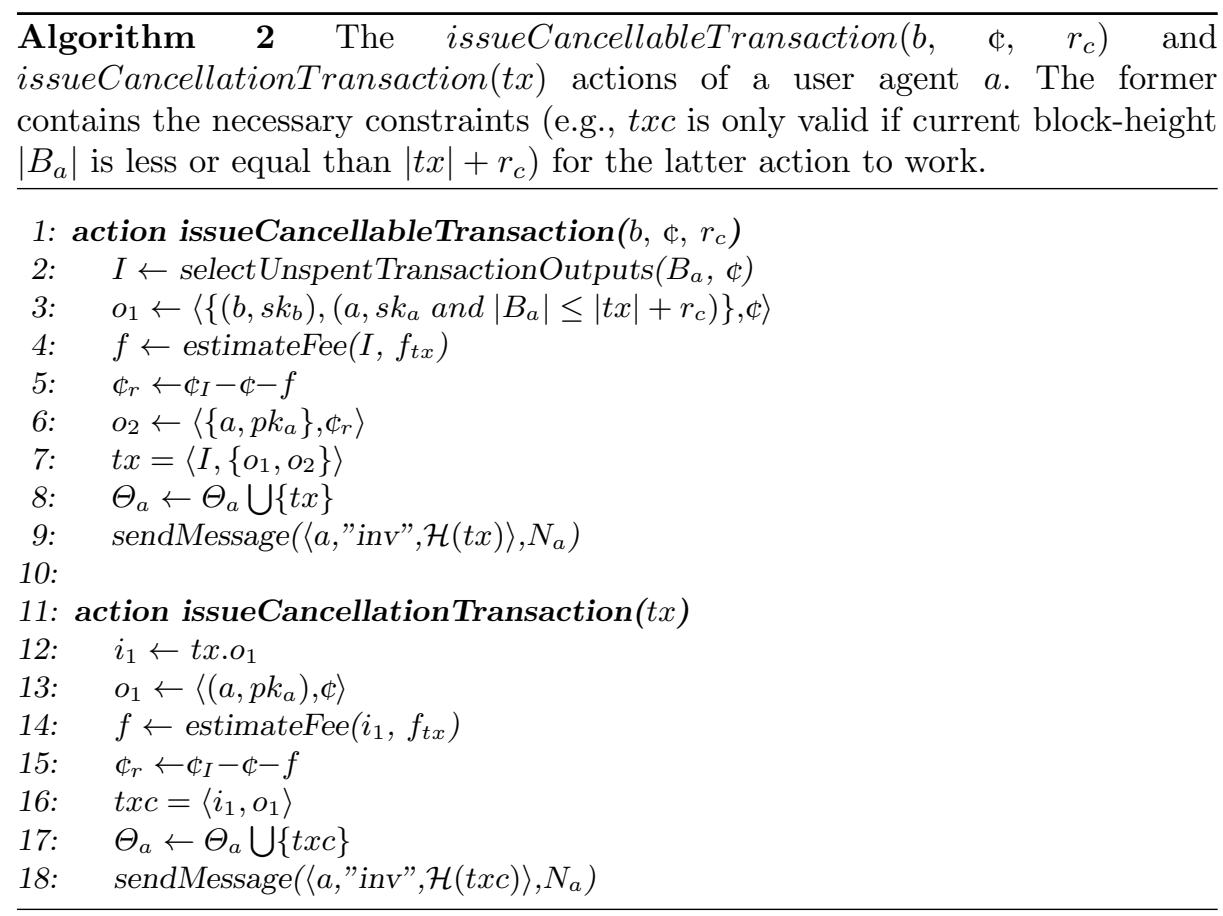

$\boldsymbol{\beta}_{2}$ : Canceling with a Replace-By-Fee (RBF) transaction User agent issues a transaction $t x r$ with a greater fee than the fee of $t x$, hoping to replace it before $t x$ hits the blockchain (i.e. $r_{c}=0$ ). Such implicitly tells miner agents to ignore $t x$. In this case, we model the reward as follows:

$$
\mathcal{R}^{\mathcal{E}}\left(\beta_{2}\right)=\left(\sum_{r=r_{0}}^{\infty}{\overline{P\left(f_{t x}\right)}}^{r-1} P\left(f_{t x}\right) \cdot\left(\sum_{s=r_{1}}^{r}{\overline{P\left(f_{t x r}\right)}}^{s-1-r_{1}} P\left(f_{t x r}\right)\right)\right) \cdot\left(\mathcal{I}-f_{t x r}\right)
$$

and its expected cost:

$$
\mathcal{C}^{\mathcal{E}}\left(\beta_{2}\right)=\sum_{r=r_{0}}^{\infty}{\overline{P\left(f_{t x}\right.}}^{r-1} \cdot\left(\sum_{s=r_{1}}^{r}{\overline{P\left(f_{t x r}\right)}}^{s-1-r_{1}} \cdot C\left(\Phi_{t x}\right)^{s-1}\right)
$$

For both, the process is analogous to extract $g_{\beta_{2}}(n)$ and $h_{\beta_{2}}(n)$, obtaining:

$$
\begin{aligned}
& g_{\beta_{2}}(n)=\left(\frac{1-x^{n}}{1-x}-y^{r_{1}} \cdot \frac{1-(x y)^{n}}{1-x y}\right) \cdot(1-x)\left(\mathcal{I}-f_{t x r}\right), \\
& \text { with } x=q_{t x}, y=q_{t x r}
\end{aligned}
$$




$$
\lim _{n \rightarrow \infty} g_{\beta_{2}}(n)=\left(\frac{1}{1-q_{t x}}-q_{t x r}^{r_{1}} \cdot \frac{1}{1-q_{t x} q_{t x r}}\right) \cdot\left(1-q_{t x}\right)\left(\mathcal{I}-f_{t x r}\right)
$$

and

$$
\begin{aligned}
h_{\beta_{2}}(n) & =\left(\frac{1-x^{n}}{(1-y)(1-x)}-y^{r_{1}} \cdot \frac{1-(x y)^{n}}{(1-y)(1-x y)}\right) \cdot d, \\
\text { with } x & =q_{t x}, y=q_{t x r} \cdot C\left(\phi_{t x}\right), d=C\left(\phi_{t x}\right)^{r_{1}},|x y|<1 \\
\lim _{n \rightarrow \infty} h_{\beta_{2}}(n) & =\left(\frac{1}{(1-y)(1-x)}-y^{r_{1}} \cdot \frac{1}{(1-y)(1-x y)}\right) \cdot d \\
& =\left(\frac{1}{\left(1-q_{t x r} \cdot C\left(\phi_{t x}\right)\right)\left(1-q_{t x}\right)}-\left(q_{t x r} \cdot C\left(\phi_{t x}\right)\right)^{r_{1}}\right. \\
& \left.\cdot \frac{1}{\left(1-q_{t x r} \cdot C\left(\Phi_{t x}\right)\right)\left(1-q_{t x} q_{t x r} \cdot C\left(\Phi_{t x}\right)\right)}\right) \cdot C\left(\phi_{t x}\right)^{r_{1}}
\end{aligned}
$$

In the next section, we will compare $\beta_{1}$ and $\beta_{2}$ by quantitatively analysing the equations formulated in this section.

\section{Analyses and Results}

We analyzed the rational behaviors of user agents proposed in Section 5.3 using gnuplot 5.2.4 $4^{15}$. It is assumed that the initial utility values $u_{0}$ of all agents are the same and high enough from the threshold $\tau$. In the following, we provide results of these analyses employing synthetic data.

Recalling Equation (4), it is clear that, depending on the values of $\overline{P(f)}$ and $C(\Phi)$, the expected value $\mathcal{E}$ may or may not converge to $-\infty$. For the geometric distributions of $\mathcal{R}^{\mathcal{E}}\left(\beta_{1}\right)$ and $\mathcal{R}^{\mathcal{E}}\left(\beta_{2}\right)$, we can see that they always converge, since all three $\overline{P\left(f_{t x}\right)}, \overline{P\left(f_{t x c}\right)} \overline{P\left(f_{t x r}\right)}$ are positive, and strictly less than 1. For the expected costs, $\mathcal{C}^{\mathcal{E}}\left(\beta_{1}\right)$ converges to the value listed in equation(13) if and only if $\left|q_{t x c} \cdot C\left(\phi_{t x}\right)\right|<1$. Analogously, $\mathcal{C}^{\mathcal{E}}\left(\beta_{2}\right)$ converges to the value of equation (19) if and only if $\left|q_{t x r} \cdot C\left(\phi_{t x}\right)\right|<1$.

Obviously, many values will have a strong impact in the results. Specifically, the correlation between the fees of a transaction $f_{t x}$ and how much they increase the probability of such transaction to be included in each round $P\left(f_{t x}\right)$. There are some online results on the average fees in Bitcoin and the average amount of blocks (rounds) a transaction takes with each fee ${ }^{16}$. Again, it is easy to see that the average amount of blocks is the expected value of a geometric distribution of which the probability $p$ is the one we are looking for, and, therefore, one can obtain this value solving the series. However, this is out of the scope of this document. Furthermore, we show further on that the cancellation transaction behavior can be better even under optimistic values for this probability, in which a small increase of the fee $f_{t x}$ incurs in a big increase in the probability $P\left(f_{t x}\right)$.

Figure 1 left shows the gain, cost, and expected values $\mathcal{E}$ of both behaviors, as functions on the number of rounds, and fixing the rest of the variables. Notice

$\overline{15}$ http://www . gnuplot.info/, last access on 24 July 2018.

16 https://bitcoinfees.earn. com/, last access on 24 July 2018. 
that here we consider that 20 Satoshis give a probability of $P(20)=0.5$, while $P(50)=0.6$. In this case, we can see how our approach is better, regardless of the round. To the right, we compare our approach with different assumptions on the increase required in the fee to increase the probability of a transaction being included. We can see how our approach is not always the best, and for example when only 10 Satoshis are required to increase the probability to $P(30)=0.6$, using a Replace-By-Fee transaction is a better behavior.

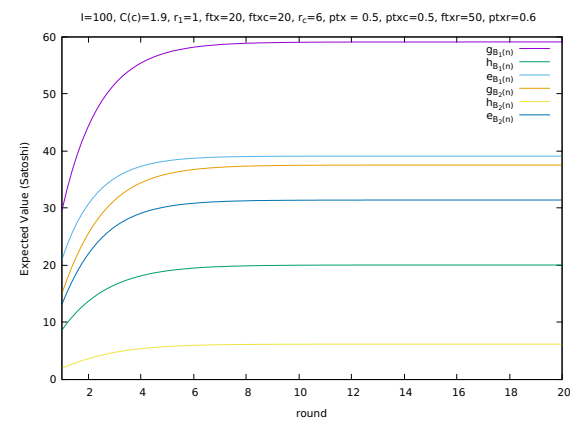

(a)

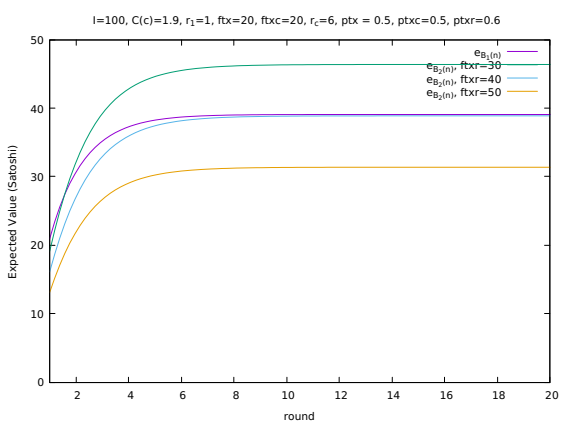

(b)

Fig. 1: Expected reward $g$, cost $h$, and value functions $e$ as functions on the number of rounds, comparing both behaviors (a). Also, comparison of different assumptions on the relationship between $f_{t x}$ and $P\left(f_{t x}\right)$ for canceling with cancellation transaction behavior $\beta_{1}$ and canceling with a RBF transaction behavior $\beta_{2}(\mathrm{~b})$.

One can note that another important variable fixed in figure 1 is $r_{1}$, that is, the round at which the user decides he wants to cancel the transaction tx, issued at round 1. In figure 1, we assume $r_{1}=1$. However, it is important to consider that the user may want to cancel the transaction later than when they issued it. Finally, $r_{c}$ can also have an impact in the results, since it increases the time in which a transaction can be cancellable by the cancellation transaction behavior $\beta_{1}$, but it also increases the cost of such behavior, since it can lead to a greater waiting time. In the following, we study how the results vary depending on $r_{1}$ and $r_{c}$.

For such cases where the series converge, we consider the values in rounds in the infinite (that is equations $(9,13,17,19)$ ), and tweak other values. Firstly, it is easy to note that behavior $\beta_{1}$ also converges for $r_{c} \rightarrow \infty$ :

$$
\begin{aligned}
\lim _{n \rightarrow \infty, r_{c} \rightarrow \infty} \mathcal{E}\left(\beta_{1}\right)\left(n, r_{c}\right) & =\lim _{n \rightarrow \infty, r_{c} \rightarrow \infty} g_{\beta_{1}}\left(n, r_{c}\right)-h_{\beta_{1}}\left(n, r_{c}\right) \\
& =\left(\mathcal{I}-f_{t x}-f_{t x c}\right)-\frac{C\left(\phi_{t x}\right)^{r_{1}}}{\left(1-q_{t x c} C\left(\phi_{t x}\right)\right)\left(1-q_{t x}\right)}
\end{aligned}
$$


While $\lim _{n \rightarrow \infty, r_{c} \rightarrow \infty} \mathcal{E}\left(\beta_{2}\right)(n)$ does not depend on $r_{c}$ by construction. It is possible to see, however, how the gain decreases for $\beta_{2}$ when $r_{1}$ increases, while in $\beta_{1}$ this is irrelevant. Nevertheless, the cost increases with $r_{c}$ for $\beta_{1}$, since this leads to higher waiting cost.

Figure 2 left compares several values depending on $r_{1}$, when the amount of rounds $n$ tends to infinity. One can see how, for $r_{c}=6$, it is a better approach to use $\beta_{1}$ for $r_{1}>4$. Furthermore, even if $r_{c}$ tends to infinity, and being optimistic in terms on the correlation between fees and probability of hitting the blockchain, $\beta_{1}$ seems to be a better behavior for $r_{1}>5$. Nevertheless, if one decides within the first rounds to cancel the transaction, then it is better to use behavior $\beta_{2}$ and issue a Replace-By-Fee transaction. Recall, however, that figure 2 plots values for $n \rightarrow \infty$. For constant number of rounds, with $r_{1}=1$, figure 1 already showed that $\beta_{2}$ is only a better approach when one can be optimistic about the probability of a transaction with a slightly higher fee hitting the blockchain.

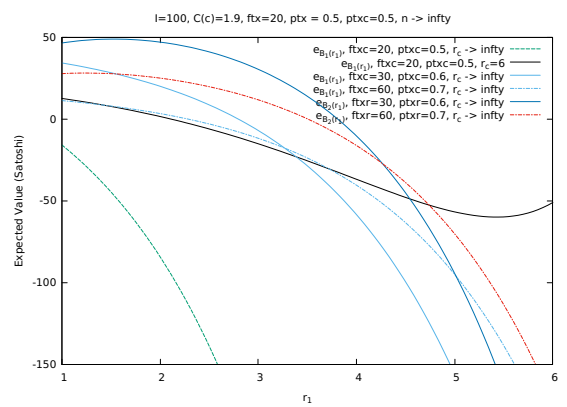

(a)

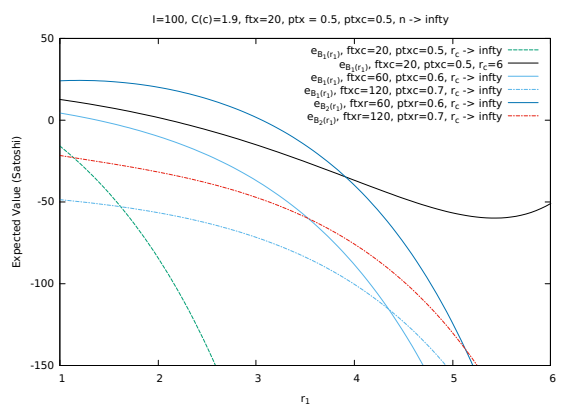

(b)

Fig. 2: Expected values for canceling with cancellation transaction behavior $\beta_{1}$ and canceling with a RBF transaction behavior $\beta_{2}$ as a function on $r_{1}$, with different values for $r_{c}, f_{t x}$ and $P\left(f_{t x}\right)$ where $\mathcal{I}=100$. To the left, optimistic values for $P\left(f_{t x}\right)$ (better for $\left.\beta_{2}\right)$.

As a result, we suggest always creating the initial transaction $t x$ as if it can be canceled by a cancellation transaction txc. However, depending on the conditions, the user may choose to issue a Replace-by-Fee transaction txr as well as trying to cancel $t x$. Nevertheless, a transaction that can be canceled in $r_{c}$ rounds leaves this transaction as non-final for the first $r_{c}$, increasing the blockdepth required for a receiver of coins to consider full ownership of such coins. The receiver can however move the coins to a new UTXO. Besides, $t x r$ is more flexible and may work even if $t x$ is prepared for being canceled by $t x c$.

In general, it seems as a good approach to firstly issue an RBF transaction, and after round $r_{1}$ issue a cancellable transaction such that $f_{t x c}+f_{t x}=f_{t x r}$. Otherwise, miners will always choose the one that gives higher reward. It is possible that the left side should be greater, $f_{t x c}+f_{t x}>f_{t x r}$, to account for 
more space used by two transactions, in such a way that the reward for the miner equalizes.

\section{Discussion}

In this section, we discuss the proposed mechanism, and the results obtained from several perspectives: fairness, security and implementability.

\subsection{Fairness}

To the best of our knowledge, this is the first study focusing on providing an explicit mechanism for cancellation of transactions to improve the fairness for users. In other words, the proposed mechanism is a first step towards guaranteeing fairness for users, given that fairness is the overall satisfaction of rational agents. Moreover, we showed that the users as rational agents have no incentive to choose the Replace-by-Fee behavior $\left(\beta_{2}\right)$ since its cancellation timeout is much less shorter. As a result, it can be said that the proposed mechanism is superior to the Replace-by-Fee workaround provided by the existing protocol.

\subsection{Security}

To avoid double-spending attacks and inconsistencies, blockchains need a selection strategy based on a predefined criteria. Bitcoin uses the longest chain strategy for selecting the main branch. Moreover, there can be situations where the network is partitioned for some time and then reconnects, with ${ }^{17}$ or without any malicious participant. The question is: which branch should be followed in case the cancellation transaction exists only in one branch, and there is another transaction that is spending the transaction we wish to cancel in another branch? Should we throw away one or more branches that creates such inconsistencies? Which branch should be followed?

In our opinion, pruning any chain (i.e. reducing the length of the chain) is dangerous and weakens its security level, which is directly proportional to the work that must be done to replace such a chain and allow an attacker to target the chain more easily. Thus, we think that the blockchain should remain append-only. Furthermore, we claim that the existing longest chain rule should remain the same. Even though with this setting the cancellation might be at times ignored, the security of the blockchain is more important than what the user desires. Furthermore, an upgrade to support cancellation of transactions should be backward compatible, not to require a hard-fork for existing Bitcoinlike blockchains.

${ }^{17}$ If there is a malicious participant that is partitioning the network, this is called a man-in-the-middle-attack. 


\subsection{Implementability}

As shown in Section 6, the proposed cancellation mechanism is feasible. This section shows its implementability in Bitcoin-like blockchains. Many such blockchains (Bitcoin, Bitcoin Cash, Litecoin, etc.) already give support to a similar feature as the one described in this document, motivated by the implementation of 2nd-layers in their network, such as the Lightning Network [16].

Bitcoin, for instance, provides support for lightning, thanks to the implementation of Bitcoin Improvement Proposal (BIP) $112^{18}$ in the system. BIP 112 implements the opcode ${ }^{19}$ OP_CHECKSEQUENCEVERIFY (typically referred to as OP_CSV), that prevents a non-final transaction from being selected for inclusion in a block until the corresponding input has reached the specified age, as measured in block-height or block-time. In this case, a non-final transaction refers to the fact that it has not reached the specified age. As such, BIP 112 already offers functionality of giving preference to some specific node to spend an UTXO.

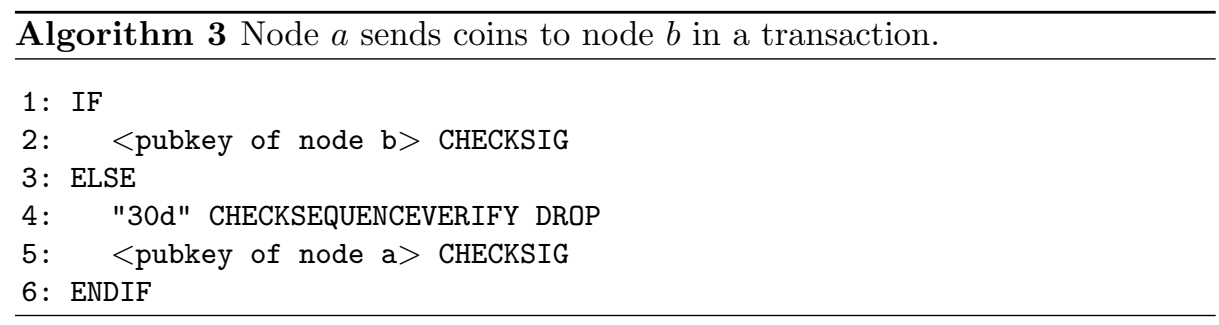

For example, node $a$ can pay node $b$ in a transaction with the redeem script given in Algorithm 3. In this case, node $b$ can spend the output at any time, while node $a$ needs to wait 30 days. After such time, any of the two can independently spend the output. However, we would like the inverse functionality where node $a$ can still cancel the issued transaction in the first 30 days, if node $b$ has not spent this output (Algorithm 4). Before 30 days, both node $a$ and node $b$ can independently spend the output. After 30 days, only node $b$ can spend it.

As illustrated in BIP $68^{20}$, and by James Prestwich ${ }^{21}$, OP_CSV compares the top stack item to the input's sequence_no field. Thus, the top stack item is parsed just as the sequence_no field for nSequence (the input-level relative time-lock). That is, it interprets 18 of the 32 bits (the remaining 14 bits are still undefined). There are two special flags: the disable and the type flag. The disable flag (bit 31 , the 32nd least significant bit) specifies that logs are disabled. The type flag

\footnotetext{
18 https://github.com/bitcoin/bips/blob/master/bip-0112.mediawiki, last access on 28 August 2018.

19 Operation codes from the Bitcoin Script language which push data or perform functions within a pubkey script or signature script.

${ }^{20}$ https://github.com/bitcoin/bips/blob/master/bip-0068.mediawiki, last access on 28 August 2018.

${ }^{21}$ https://prestwi.ch/bitcoin-time-locks/, last access on 28 August 2018.
} 


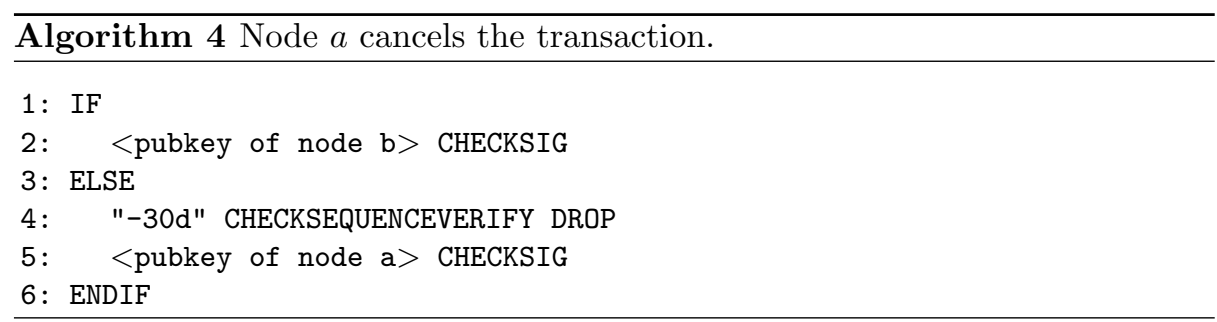

(bit 22, the 23rd least significant bit) specifies the type of information: if set, the remaining 16 least significant bits are interpreted in units of 512 seconds granularity, if not, they are interpreted as block-height. The flag $(1 \leq \leq 22)$ is the highest order bit in a 3-byte signed integer for use in bitcoin scripts as a 3-byte PUSHDATA with OP_CHECKSEQUENCEVERIFY (BIP 112), as detailed in the specification of BIP 68.

At the time of writing, using OP_CSV with value 0x00400001 and 0x00C00001 applies the same timelock: a relative locktime of 512 seconds. Moreover, the specification says OP_CSV "errors if the top stack item is less than 0". We propose, however, to consider the sign bit as the sign flag, and interpret instead this negative value exactly as detailed in the aforementioned example.

OP_CSV is useful for considering a relative time from the inclusion of the transaction in the blockchain. If, instead, one would like to specify an absolute time, or a block-height, this is possible using OP_CHECKLOCKTIMEVERIFY (OP_CLTV) (see BIP $65^{22}$ ). Analogously, we propose the same consideration for OP_CLTV. These two simple backward compatible features can be implemented after proposing them in a new BIP. Also, for simplicity, and to comply with the current definition of OP_CSV and OP_CLTV, we propose referring to each upgrade as OP_CHECKSEQUENCEVERIFYINVERSE (OP_CSVI) and OP_CHECKLOCKTIMEVERIFYINVERSE (OP_CLTVI).

As such, we show that it is possible, and convenient, to implement the required opcodes to give support for cancellation transactions in Bitcoin, making use of the sign bit, that was useless until now, although it was respected as the sign bit. We do this in a backward compatible way.

\section{Conclusions}

In this paper, we proposed a novel explicit transaction cancellation mechanism that cancels issued transactions under certain conditions. Such a mechanism increases the fairness for the users and thus increases the security and sustainability of the blockchain system. To avoid security issues and related complex analyses, the proposed mechanism sticks as much as possible to the original Bitcoin protocol, introducing mechanisms to improve the degree of fairness of the

${ }^{22}$ https://github.com/bitcoin/bips/blob/master/bip-0065.mediawiki, last access on 28 August 2018. 
system. To this end, we showed the implementation of our approach for Bitcoin, and consider also its implementability for Bitcoin-like blockchains.

\section{References}

1. Asayag, A., Cohen, G., Grayevsky, I., Leshkowitz, M., Rottenstreich, O., Tamari, R., Yakira, D.: Helix: A scalable and fair consensus algorithm. Tech. rep., Orbs Research (2018), https://orbs.com/wp-content/uploads/2018/07/ Helix-Consensus-Paper-V1.2-1.pdf

2. Asokan, N.: Fairness in electronic commerce (1998)

3. Buchman, E.: Tendermint: Byzantine Fault Tolerance in the Age of Blockchains. Ph.D. thesis, University of Guelph (6 2016)

4. Carlsten, M., Kalodner, H., Weinberg, S.M., Narayanan, A.: On the instability of bitcoin without the block reward. In: Proceedings of the 2016 ACM SIGSAC Conference on Computer and Communications Security. pp. 154-167. ACM (2016)

5. Eyal, I., Sirer, E.G.: Majority is not enough: Bitcoin mining is vulnerable. In: Int. Conf. on Fin. Cryp. and Data Security. pp. 436-454. Springer (2014)

6. Garay, J., Kiayias, A., Leonardos, N.: The Bitcoin Backbone Protocol: Analysis and Applications, pp. 281-310. Springer Berlin Heidelberg, Berlin, Heidelberg (2015), http://dx.doi.org/10.1007/978-3-662-46803-6_10

7. Gürcan, Ö., Del Pozzo, A., Tucci-Piergiovanni, S.: On the bitcoin limitations to deliver fairness to users. In: Panetto, H., Debruyne, C., Gaaloul, W., Papazoglou, M., Paschke, A., Ardagna, C.A., Meersman, R. (eds.) On the Move to Meaningful Internet Systems. OTM 2017 Conferences. pp. 589-606. Springer Int. Publishing, Cham (2017)

8. Herlihy, M., Moir, M.: Enhancing accountability and trust in distributed ledgers. CoRR abs/1606.07490 (2016), http://arxiv.org/abs/1606.07490

9. Kwon, J.: Tendermint: Consensus without mining. Tech. rep., Tendermint (2014), https://tendermint.com/static/docs/tendermint.pdf

10. Liu, J., Li, W., Karame, G.O., Asokan, N.: Towards fairness of cryptocurrency payments. arXiv preprint arXiv:1609.07256 (2016)

11. Merkle, R.C.: A Digital Signature Based on a Conventional Encryption Function, pp. 369-378. Springer Berlin Heidelberg, Berlin, Heidelberg (1988)

12. Miller, A., Xia, Y., Croman, K., Shi, E., Song, D.: The honey badger of bft protocols. In: Proceedings of the 2016 ACM SIGSAC Conference on Computer and Communications Security. pp. 31-42. CCS '16, ACM, New York, NY, USA (2016), http://doi.acm.org/10.1145/2976749.2978399

13. Nakamoto, S.: Bitcoin: A peer-to-peer electronic cash system (2008), https:// bitcoin.org/bitcoin.pdf

14. Pappalardo, G., di Matteo, T., Caldarelli, G., Aste, T.: Blockchain inefficiency in the bitcoin peers network. CoRR abs/1704.01414 (2017), http://arxiv.org/abs/ 1704.01414

15. Pass, R., Seeman, L., Shelat, A.: Analysis of the blockchain protocol in asynchronous networks. IACR Cryptology ePrint Archive 2016, 454 (2016)

16. Poon, J., Dryja, T.: The bitcoin lightning network. i pp. 1-22 (2015)

17. Russell, S.J., Norvig, P.: Artificial Intelligence - A Modern Approach (3. internat. ed.). Pearson Education (2010)

18. Sapirshtein, A., Sompolinsky, Y., Zohar, A.: Optimal selfish mining strategies in bitcoin. In: International Conference on Financial Cryptography and Data Security. pp. 515-532. Springer (2016) 